\title{
Leak after Laparoscopic Cholecystectomy; Incidence and Management
}

\author{
Alaa Abbas Sabry, Mohamed Gamal El Fouly, Mohamed Walaa El Dien Fawzy \\ Department of General Surgery, Faculty of Medicine, Ain Shams University \\ Corresponding author: Mohamed Walaa El Dien, E-Mail: mohamed_walaa7@ hotmail.com, Mobile: 01062041513
}

\begin{abstract}
Background: laparoscopic cholecystectomy is currently the procedure of choice for symptomatic gallstones. It has evolved from an innovative, but time-consuming, novelty to a routine day-case procedure over the last 20 years.

Purpose: to estimate the incidence and management options of post laparoscopic cholecystectomy biliary leaks. Also, it is a trial to advocate a minimally invasive structured management protocol to treat patients with bile leak.

Patients and Methods: this study was a case series of 100 patients who underwent laparoscopic cholecsystectomy. We didn't include patients who had: Biliary leak post Laparoscopic converted to open cholecystectomy. Biliary leak due to other procedures. Biliary leaks managed by open surgery. Biliary injuries discovered and definitively managed intra-operatively. The included patients presented to the General Surgery Department at Manshyt El Bakry General Hospital. Patients' age ranged from 12 to 65 years and patients' sex was distributed as 78 females and 22 males.

Results: in our cases there were two out of the 100 patients suffered from biliary leak ,one of the two cases that were presented with biliary leak after surgery was due to direct injury to accessory duct of Luschka and the other one was due to a slipped clips.

Conclusion: cholecystectomy is the most frequently performed abdominal operation in the United States, with currently over 700,000 cases occurring per year. Bile leak as a result of cholecystectomy is uncommon. The frequency in large series is less than $2 \%$ and in our study it was exactly $2 \%$. And the treatment of that leak varies from conservative treatment to ERCP and stent insertion like we did.
\end{abstract}

Keywords: Laparoscopic Cholecystectomy, Endoscopic Biliary Sphincterotomy, Endoscopic Retrograde Cholangiopancreatography

\section{INTRODUCTION}

There have been several prospective, randomized trials comparing open cholecystectomy with laparoscopic cholecystectomy and results have been mixed in demonstrating advantages of laparoscopic over comparing open cholecystectomy in treating elective symptomatic cholelithiasis. The largest study prospectively randomized 310 patients to laparoscopic versus mini-laparotomy comparing open cholecystectomy with 155 patients in each group. Conversion to large-incision cholecystectomy was significantly more common with laparoscopic (13\% vs. $4 \%$ ) and complications were significantly more frequent with laparoscopic (9\% vs. 3\%). When laparoscopic was successful, there was no significant cost difference between the two procedures ${ }^{(1)}$.

Though bile leak after laparoscopic cholecystectomy is uncommon but can occur in $0.3-2.7 \%$ of patients, it is defined as the persistent leakage of bile from the biliary tree. This can arise from an injury to the common bile or hepatic duct but it is generally accepted that the vast majority arise from the cystic duct stump or a sub-vesical duct of Luschka ${ }^{(2,3)}$.

A bile leak may result in a biliary fistula, a subhepatic/ subphrenic collection and localized or generalised peritonitis. Clearly, this can be associated with significant morbidity and even mortality, particularly if it is not identified and treated at an early stage ${ }^{(4)}$.

In the early 1990s when both laparoscopic cholecystectomy and minimally invasive techniques were in their infancy, bile leaks were managed conservatively; if the patient did not improve, a laparotomy was often performed. Management was anecdotal and based upon the experience of the surgeon. However, with the advantage of improved Radiological percutaneous drainage, therapeutic endoscopic retrograde cholangiography (ERC), and increased confidence with laparoscopic techniques including suturing, it became clear that bile leaks could be managed in a minimally invasive manner, potentially reducing morbidity and mortality. Prompt access to the full range of techniques is important, as is a structured approach ${ }^{(5)}$.

\section{AIM OF WORK}

Is to estimate the incidence and management options of post laparoscopic cholecystectomy biliary leaks. Also, it is a trial to advocate a minimally invasive structured management protocol to treat patients with bile leak. 


\section{PATIENTS AND METHODS}

Patients' population: This study is a case series of 100 patients who underwent laparoscopic cholecsystectomy.

We didn't include patients who had: Biliary leak post Laparoscopic converted to open cholecystectomy. Biliary leak due to other procedures. Biliary leaks managed by open surgery. Biliary injuries discovered and definitively managed intra-operatively. The included patients presented to the General Surgery Department at Manshyt El Bakry General Hospital in Cairo, Egypt. Patients' age ranged from 12 to 65 years and patients' sex was distributed as 78 females and 22 males. (78\%vs $22 \%$ ). Clinically all the patients were presented to under go laparoscopic cholecystectomy operation and post operative follow up for any evidence of biliary leak, laboratory and radiological investigations were carried out in order to detect the causes of biliary leak if there was biliary leak, grade of injury and the ways to manage.

Ethical considerations: After obtaining the hospitals Research/Ethics Committee approval and written informed consents from the patients, this study will be carried on.

\section{Patients' evaluation:}

Clinical examination: Complete history taking. Detailed general and local physical examination. Operative details were all reviewed.

Laboratory investigations: Full blood count. AST. ALT. Alkaline phosphatise. Direct bilirubin. Total bilirubin.

\section{RESULTS}

From the 100 patients in this study, we had two cases of biliary leakage after laparoscopic cholecystectomy.

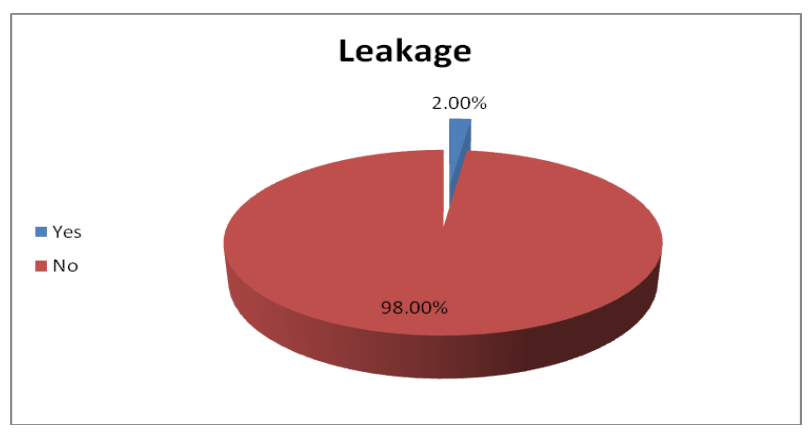

Figure (1): Percentage of incidence.
In our case both of the cases that were presented with biliary leak after the surgery one was apparent after 2 days and after 1 day of the surgery.

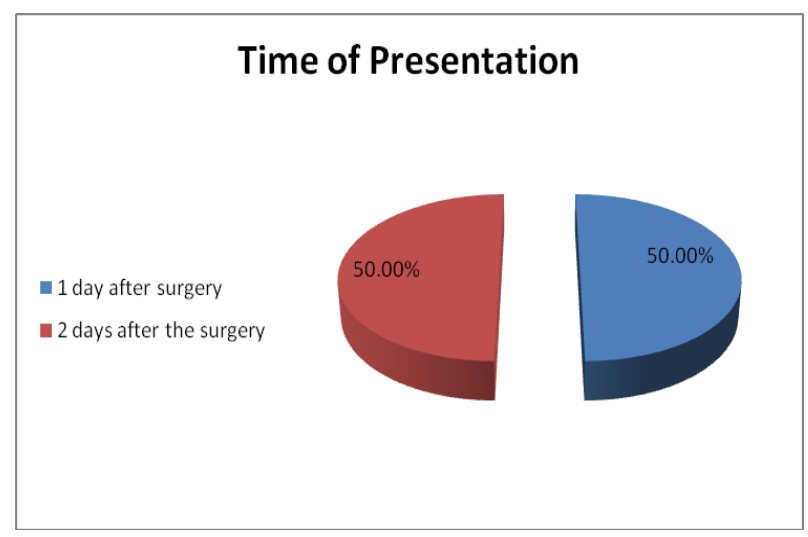

Figure (2): Time of presentation of biliary leak.

The same patient showed a bile collection detected by pelvi-abdominal US and CT abdomen, and showed that the leak was due to slipped clip.

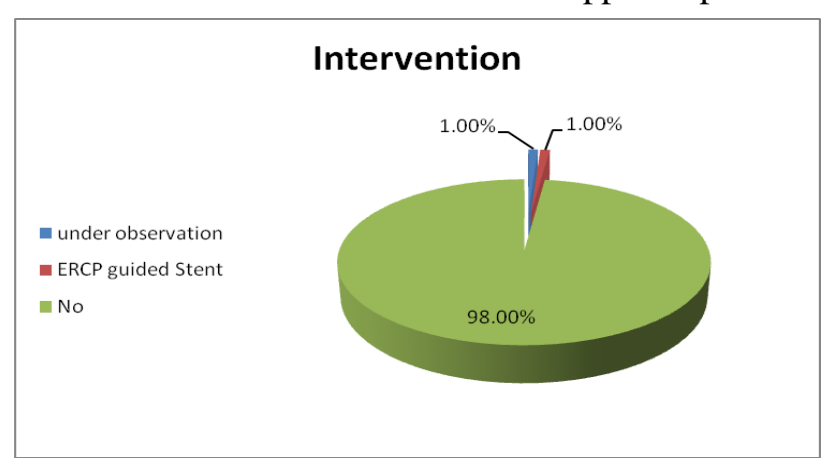

Figure (3): Intervention done to patients with biliary leak.

In our series patients were discharged home within 10-12 days after intervention, all showed cessation of bile leak and treatment from sepsis prior to discharge, While other patients from 1-3 days.

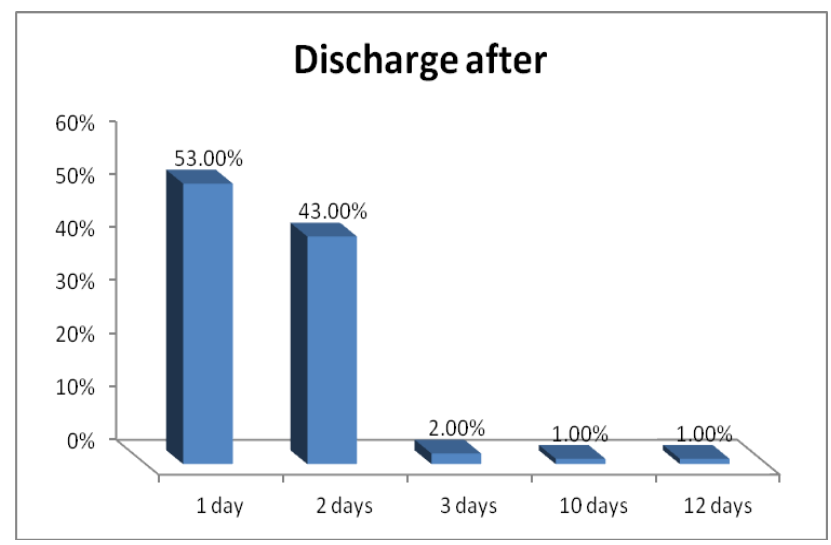

Figure (4): Time of discharge after surgery. 


\section{DISCUSSION}

Laparoscopic cholecystectomy is currently the procedure of choice for symptomatic gallstones. It has evolved from an innovative, but time-consuming, novelty to a routine day-case procedure over the last 20 years ${ }^{(1)}$.

Similarly, the management of bile leaks following this procedure has changed. However, the fundamental principles underpinning this have not, i.e. successful drainage of a bile leak is critical. If drainage is inadequate, sepsis and biliary peritonitis develop and this remains a clear indication for surgical intervention.

The introduction of both ERC and relaparoscopy as opposed to laparotomy are the two major differences in management being recently established many specialized centres, ERCP has both a diagnostic and therapeutic roles, It allows identification of both the site of the leak as well as any residual stones within the bile duct that may be contributing to it. Such stones can be removed and various strategies used to reduce the pressure gradient between the bile duct and the duodenum created by contraction of the sphincter of Oddi. This encourages the preferential flow of bile into the duodenum thus attenuating the bile leak and allowing the site to heal. Indeed, this, in effect, means that one no longer has to perform an exploratory laparotomy with the aim of finding and closing the leak ${ }^{(6)}$.

In our case series we identified 100 patients, 78 females and 22 males with age range between 12 and 65 years.

Though Observational studies have suggested a larger number of bile duct injuries with early surgery but this was not evident from the randomized trials. In a Meta-analysis of randomized controlled trials on the safety and effectiveness of early versus delayed laparoscopic cholecystectomy for acute cholecystitis done by Gurusamy et al. ${ }^{(7)}$, they looked into five trials with 451 patients. There was no significant difference between the two groups in terms of bile duct injury. The trials reported bile duct injury requiring reoperation.There was no significant difference between the two groups with respect to this complication. The bile duct injury rate was 0.5 per cent (one of 222) in the early group versus 1.4 per cent (three of 216) in the delayed group ${ }^{(7)}$.

In our case one of the two cases that were presented with biliary leak after surgery was due to direct injury to accessory duct of Luschka.

In 20 to $50 \%$ of patients the Biliary Duct injuries may be recognized at the time of surgery and can be repaired immediately, More often the injury becomes apparent in a delayed fashion, weeks to months or even years later Most bile duct injuries present between 3 and 32 days after the surgery but some have presented as late as postoperative day, The symptoms and signs of delayed presentation are typically nonspecific, with abdominal pain being the most common, Other common clinical presentations include general malaise, liver function test values indicative of cholestasis, jaundice, fever, and sepsis. The nature of the clinical picture is related to the pathologic condition prevailing at the time of presentation, namely bile leak or stasis/obstruction.

In our case both of the cases that were presented with biliary leak after the surgery one was apparent after 2 days and after 1 day of the surgery.

In a systematic review of intra-operative cholangiography in cholecystectomy identified eight randomized trials were including 1715 patients. Six trials assessed the value of routine intra-operative cholangiography in patients at low risk of choledocholithiasis. Two trials randomized all patients (including those at high risk) to routine or selective intraoperative cholangiography. Two cases of major bile duct injury were reported, and 13 of retained CBD stones. No trial demonstrated a benefit in detecting CBD stones. Intra-operative cholangiography added a mean of $16 \mathrm{~min}$ to the total operating time. They finally concluded that there is no robust evidence to support or abandon the use of intra-operative cholangiography to prevent retained CBD stones or bile duct injury. Level 1 evidence for intra-operative cholangiography is of poor to moderate quality. None of the trials, alone or in combination, was sufficiently powered to demonstrate a benefit of intra-operative cholangiography ${ }^{(8)}$.

A number of techniques have been proposed to manage bile duct leaks including endoscopic sphincterotomy alone, nasobiliary tube drainage and internal biliary-duodenal stent Endoscopic sphincterotomy alone does not appear as effective as the latter two approaches and increases the risk of ERCrelated complications, Furthermore, unless stone extraction is planned, it is unusual for a sphincterotomy to be necessary in order to insert either nasobiliary or internal stents. However, nasobiliary tubes are not 
particularly well tolerated by patients and can easily become displaced ${ }^{(1)}$.

Neither diagnostic nor therapeutic ERC were used routinely. Thus, a common indication for surgical intervention was persistence of the bile leak, in comparison, following introduction of the minimally invasive protocol, this was the main indication for ERC and stenting but was not an indication for surgical intervention ${ }^{(6)}$.

In our study one case underwent ERCP with sphincterotomy was done and stent was inserted.

The same patient showed a bile collection detected by pelvi-abdominal US and CT abdomen, and showed that the leak was due to slipped clip.

Kaffes et al. ${ }^{(9)}$ showed in their retrospective study on 100 patients presented with bile leak post laparoscopic cholecystectomy, ERCP was performed and a cholangiogram was obtained in 96 patients. In 4 patients, cholangiography could not be obtained for technical problems. ERCP demonstrated a leak in 80 $(83 \%)$ of patients. Of the 16 in whom a leak was not identified, 3 had a major bile-duct injury, two had complete duct obstruction because of a surgical clip, and one had a complete transection of the right hepatic duct. The most common site of the leak was the cystic duct stump followed by ducts of Luschka Stent insertion was the most common intervention A 10 Fr stent was placed in 57 patients, and a $7 \mathrm{Fr}$ stent was placed in 15 patients, the latter primarily those in whom a sphincterotomy was not performed. In 6 patients, no leak was identified and no intervention was performed .

On an intention-to-treat basis, endoscopic intervention at ERCP had a success rate for resolution of the leak of $92 \%(92 / 100)$. If the 3 cases of major bile-duct injury are excluded, the success rate was 95\% (92/97). Of the 80 patients with a demonstrated leak, 76 were successfully treated $(95 \%)$. The median time for resolution of the leak was 3 days (range 1-39 days). They noted that the number of patients in whom further intervention was required was significantly higher in the sphincterotomy alone group compared with the stent alone group, or with the stent and sphincterotomy group $(\mathrm{p}<0.01)$. When the 3 patients with major bile-duct injury are excluded, the need for surgery to control the leak subsequent to ERCP is comparable for all endoscopic treatment groups except sphincterotomy alone, which was associated with significantly more surgical procedures $(\mathrm{p}=0.001$, Fisher exact test).
One asymptomatic patient in the stent alone group had an ongoing leak detected at follow-up ERCP 4 weeks after stent insertion. The stent was exchanged, and, at ERCP 6 weeks later, the leak was closed. Three patients ( 1 treated by stent alone, 2 sphincterotomy alone) required repeat ERCP to control an ongoing leak. Stent insertion in the two patients treated initially by sphincterotomy alone resulted in closure of the leak. The patient treated initially by stent alone (10 Fr) had a new stent inserted after a sphincterotomy and bile-duct stone extraction; the leak resolved without sequelae ${ }^{(10)}$.

They concluded that optimal endoscopic treatment for a simple bile-duct leak is insertion of a straight plastic stent at least $7 \mathrm{Fr}$ in diameter. The stent should be removed after 4 weeks, and, in the majority of cases, follow-up cholangiography will not be required, particularly for uncomplicated cystic-duct stump or peripheral duct of Luschka leaks. However, cholangiography should be obtained if there is known or probable stricture formation, for example, if the leak had arisen directly from the bile duct or the right hepatic duct. The decision for a follow-up ERCP, as opposed to stent removal alone, has both ethical implications and economic considerations ${ }^{(10)}$.

From the endoscopists stand point, biliary injuries during laparoscopic cholecystectomy can result in bile leaks, biliary stricture formation, or both.

\section{Bile leaks are divided into:}

1. Low grade (LG), where the leak can only be identified after complete opacification of the intrahepatic biliary system.

2. High grade $(\mathrm{HG})$, where the leak can be observed before intrahepatic opacification.

Sandha et al. ${ }^{(11)}$ proposed an algorithm for their management of post laparoscopic cholecystectomy bile leaks based on the grade of leak in a non-randomized setting. They concluded that endoscopic biliary sphincterotomy (EBS) alone without stent placement is a viable treatment option for most patients with low grade leaks, unless there is a compelling indication for stent insertion such as retained stone, biliary injury with stricture formation, coagulopathy precluding EBS, or sepsis necessitating immediate closure of the leak. All high grade lesions were successfully treated with EBS and stent placement. The study did not address whether EBS alone had an equally 
favourable outcome as biliary stent placement in patients with low grade leaks.

Mavrogiannis et al. ${ }^{(12)}$ compared biliary stenting alone vs. EBS plus biliary stenting in a prospective randomized study limited to patients with endoscopically-proven post laparoscopic cholecystectomy bile leaks (PCBL), they concluded that both methods were equally efficacious in resolving low grade leaks and did not differ significantly in their adverse effect profile. However, it is known that EBS is associated with both shortand long-term adverse consequences ${ }^{(12)}$.

Mild pancreatitis is perhaps the most common early complication followed by hemorrhage, perforation and sepsis, Long-term effects include papillary stenosis and cholangitis, the authors therefore recommended against routine EBS in the management of patients with low grade bile leaks ${ }^{(10)}$.

In the same study, 7 Fr plastic biliary stents were placed due to ease of insertion in the group without EBS. In the group with EBS, however, wider $10 \mathrm{Fr}$ stents were used to minimize risk of migration. It is worth mentioning that $10 \mathrm{Fr}$ stents could have been used in both groups, since there is no evidence that insertion of wider $10 \mathrm{Fr}$ stents is associated with any increased risk of complications. In fact, it may pose a lower risk of stent clogging in the setting of bile leak with associated choledocholithiasis ${ }^{(13)}$.

There is also debate regarding the optimal proximal extent of the biliary stent. Some investigators believe that the endoprosthesis should extend beyond the site of leakage ${ }^{(14)}$.

Others propose that placement of a transpapillary stent in an effort to equilibrate pressure across the biliary tree and duodenum is sufficient to stop the leak ${ }^{(15)}$.

Actually, based on the Young-Laplace-Gauss equation, the shorter in length and larger in width the endoprosthesis, the lower the pressure gradient across the papilla. In the study by Ryan et al. ${ }^{(16)}$ there was no impact of proximal stent position on outcome after stent placement in the setting of post laparoscopic cholecystectomy bile leaks ${ }^{(16)}$.

There seems to be consensus with respect to the duration of stent placement. Successful endotherapy leads to symptom resolution in most patients within the first week and the stent remains in place for a period of 6 weeks. This seems to be of sufficient duration for biliary leak healing in most studiesfollowing this period, the biliary stents can be removed by simple endoscopy with the use of a snare or stent-retrieving forceps ${ }^{(17)}$.

As far as high grade leaks are concerned, there are recent reports in the literature supporting the placement of fully covered removable selfexpandable metal stents (SEMS) with successful outcomes ${ }^{(18)}$

In another experience Nawaz et al. ${ }^{(19)}$ in a small series of patients patients with high grade leaks secondary to incomplete cholecystectomies, refractory to $10 \mathrm{Fr}$ biliary stenting, all patients were successfully treated with placement of 8 or $10 \mathrm{~mm}$ in diameter fully covered SEMS long enough to cover the cystic duct take-off ${ }^{(19)}$.

The featured study in this issue of Annals of Gastroenterology by Fasoulas et al. ${ }^{(20)}$ is an 11-year retrospective review of all patients presenting at a tertiary care center in Northern Greece with a suspected diagnosis of PCBL. It is interesting to note that all patients underwent EBS, stone extraction when indicated, and placement of wide $10 \mathrm{Fr}$ or $11.5 \mathrm{Fr}$ stent extending across the site of bile leak ${ }^{(20)}$.

In case of LG leaks, such as those originating from the duct of Luschka, short $(5 \mathrm{~cm}$ in length) transpapillary stents were placed. Overall, endoscopic intervention was highly successful in treating bile leaks in $98 \%$ (65 out of 66 patients). The most common ERCP complications encountered included post-ERCP pancreatitis, intraprocedural bleeding and biliary stricture. The authors' results from this series are in accordance with the published literature. They suggested enhanced vigilance and early ERCP so as to initiate diagnostic and therapeutic interventions in patients with diffuse abdominal pain, fever, malaise, abnormal liver injury tests, and increased output from surgical drain postcholecystectomy. This would ultimately result in reduced hospital stay and total costs ${ }^{(20)}$.

In our series patients were discharged home within 10-12 days after intervention, all showed cessation of bile leak and treatment from sepsis prior to discharge, While other patients from 1-3 days.

\section{CONCLUSION}

There are several possible treatment strategies for such leaks. A large body of data 
supports the early use of ERCP to exclude significant bile-duct injury and to effect closure of the leak by various endoscopic and minimally invasive means. There are several possible treatment strategies for such leaks. A large body of data supports the early use of ERCP to exclude significant bile-duct injury and to effect closure of the leak by various endoscopic and minimally invasive means. This algorithm, we believe, will guide the surgeon for management of patients presented with post laparoscopic cholecystectomy bile leak.

\section{REFERENCES}

1. McGinn FP, Miles AJ, Uglow $M$ et al. (2001): Randomized trial of laparoscopic cholecystectomy and mini-cholecystectomy. Br J Surg., 82: 1374-1377.

2. Vecchio R, MacFadyen BV and Latteri $S$ (2000): Laparoscopic cholecystectomy: an analysis of 114,005 cases of United States series. Int Surg., 83: 215-9.

3. McMahon AJ, Fullarton G, Baxter JN et al. (2004): Bile duct injury and bile leakage in laparoscopic cholecystectomy. Br J Surg., 82: 307-13.

4. Buanes $\mathrm{T}$, Waage $\mathrm{A}$, Mjaland $\mathrm{O}$ et al. (2006): Bile leak after cholecystectomy significance and treatment. Results from the National Norwegian Cholecystectomy Registry. Int Surg., 81: 276-9.

5. De Palma GD, Galloro G, Iuliano G et al. (2002): Leaks from laparoscopic cholecystectomy. Hepatogastroenterology , 49: 924-5.

6. Leeder PC, Mathews T, Krzeminska $\mathrm{K}$ et al. (2004): Routine day-case laparoscopic cholecystectomy. Br J Surg., 91: 312-317.

7. Gurusamy K, Junnarkar S, Farouk M et al. (2008): Meta-analysis of randomized controlled trials on the safety and effectiveness of day-case laparoscopic cholecystectomy. British Journal of Surgery, 95(2): 161-8.

8. Ford JA, Soop M, Du J et al. (2012): Systematic review of intraoperative cholangiography in cholecystectomy, British Journal of Surgery, 99: 160-167.

9. Kaffes AJ, Hourigan L, De Luca N et al. (2005): Impact of endoscopic intervention in 100 patients with suspected postcholecystectomy bile leak. Gastrointestinal Endoscopy, 61(2): 269-75.
10. Freeman ML, Nelson DB, Sherman S, et al. (2002): Complications of endoscopic biliary sphincterotomy. N Engl J Med., 335: 909-918.

11. Sandha GS, Bourke MJ, Haber GB et al. (2004): Endoscopic therapy for bile leak based on a new classification: results in 207 patients. Gastrointestinal Endoscopy, 60(4): 567-74.

12. Mavrogiannis $\mathrm{C}$, Liatsos $\mathrm{C}$, Papanikolaou IS et al. (2006): Biliary stenting alone versus biliary stenting plus sphincterotomy for the treatment of post-laparoscopic cholecystectomy biliary leaks: a prospective randomized study. Eur J Gastroenterol Hepatol., 18: 405-409.

13. Bertrand C. Prevalence of bile duct injury following cholecystectomy. Acta Chir Belg., 103-111.

14. Lillemoe KD (2011): Biliary Injuries, strictures and Sclerosing Cholangitis, Greenfield, https:// accesssurgery. mhmedical. com/ content. aspx?bookid $=429 \&$ sectionid

15. Lillemoe KD, Pitt HA and Cameron JL (2000): Postoperative bile duct strictures. Surg Clin North Am., 70: 1363.

16. Ryan ME, Geenen JE, Lehman GA et al. (2000): Endoscopic intervention for biliary leaks after laparoscopic cholecystectomy: a multicenter review. Gastrointest Endosc., 47: 261-266.

17. Bjorkman DJ, Carr-Locke DL, Lichtenstein DR et al. (2005): Postsurgical bile leaks: endoscopic obliteration of the transpapillary pressure gradient is enough. Am J Gastroenterol., 90: 2128-2133.

18. Wang AY, Ellen K, Berg CL et al. (2009): Fully covered self-expandable metallic stents in the management of complex biliary leaks: preliminary data - a case series. Endoscopy, 41: 781-786.

19. Nawaz $H$ and Papachristou GI (2011): Endoscopic treatment for postcholecystectomy bile leaks: update and recent advances. Annals of Gastroenterology: Quarterly Publication of the Hellenic Society of Gastroenterology, 24(3): 161.

20. Fasoulas $\mathrm{K}$, Zavos $\mathrm{C}$, Chatzimavroudis $\mathrm{G}$ et al. (2011): Eleven-year experience on the endoscopic treatment of post-cholecystectomy bile leaks. Ann Gastroenterol., 24: 200-205. 